\title{
In vitro evaluation of microleakage under orthodontic brackets bonded with different adhesive systems
}

\author{
Ramin Atash ${ }^{1}$, Ali Fneiche ${ }^{1}$, Sibel Cetik ${ }^{2}$, Babak Bahrami ${ }^{1}$, Alain Balon-Perin ${ }^{1}$, \\ Maria Orellana ${ }^{1}$, Régine Glineur ${ }^{1}$
}

Correspondence: Dr. Sibel Cetik

Email: sibel.cetik@ulb.ac.be

\begin{abstract}
'Department of Stomatology and Dentistry, Erasmus Hospital, Université Libre de Bruxelles, Brussels, Belgium,

${ }^{2}$ Department of Stomatology and Dentistry, Erasmus Hospital and Laboratory of Physiology and Pharmaceutics, Faculty of Medicine, Université Libre de Bruxelles, Brussels, Belgium
\end{abstract}

\section{ABSTRACT}

Objective: Adhesives systems have a drawback when utilized for bonding orthodontic brackets: they shrink during photopolymerization creating microleakage. The aim of this study was to assess the stability of different orthodontic adhesives around brackets and enamel. Materials and Methods: Sixty noncarious mandibular premolars extracted for orthodontic reasons were randomly divided into six groups of adhesives used for bonding brackets to dental enamel: NeoBond ${ }^{\circledR}$ Light Cure Adhesive Kit, Transbond ${ }^{\mathrm{TM}}$ Plus Self-Etching, Victory V-Slot APC PLUS ${ }^{\circledR}+$ Transbond $^{\mathrm{TM}}$ MIP, Rely-A-Bond ${ }^{\circledR}$ Kit, Light Cure Orthodontic Adhesive Kit $\left(\mathrm{OptiBond}^{\mathbb{}}\right)$, and Transbond ${ }^{\mathrm{TM}}$ MIP. Following bonding, all teeth underwent 2500 cycles of thermal cycling in baths ranging from $5^{\circ} \mathrm{C}$ to $55^{\circ} \mathrm{C}$ before being immersed in $2 \%$ methylene blue for $24 \mathrm{~h}$. All samples were examined under a binocular microscope to assess the degree of microleakage at the "bracket-adhesive" and "adhesive-enamel" interfaces in the gingival and occlusal regions of the bracket. Results: A significant difference was found at the "occlusal bracket-adhesive" interface. The highest microleakage values were found in the occlusal region, although no significant. Microleakage was observed in all groups. Conclusion: Group 2 had the highest microleakage values whereas Group 6 had the lowest values.

Key words: Adhesive, bracket bonding, microleakage, shrinkage

\section{INTRODUCTION}

There are several methods to prepare enamel for bonding: self-etching, which combines an etching acid and a primer in one single step, thereby reducing procedure time; "classic" etching, which involves applying a strong acid onto the enamel surface, followed by the application of a primer and a bonding agent. Another procedure is laser conditioning of the

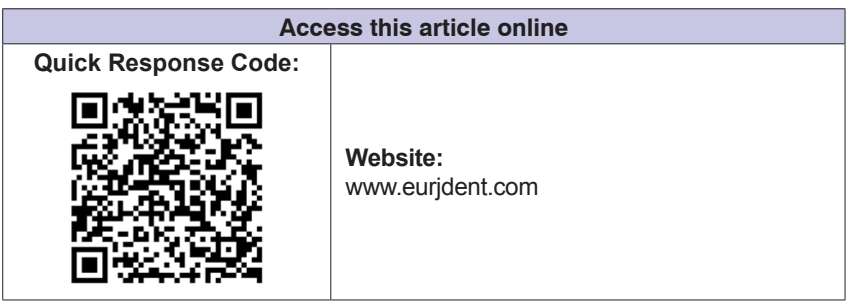

enamel, which is performed at a specified frequency and duration..$^{[1-6]}$

One of the main drawbacks of adhesive materials is polymerization shrinkage during curing, which creates a space between the adhesive and the enamel

This is an open access article distributed under the terms of the Creative Commons Attribution-NonCommercial-ShareAlike 3.0 License, which allows others to remix, tweak, and build upon the work non-commercially, as long as the author is credited and the new creations are licensed under the identical terms.

For reprints contact: reprints@medknow.com

How to cite this article: Atash R, Fneiche A, Cetik S, Bahrami B,
Balon-Perin A, Orellana M, et al. In vitro evaluation of microleakage
under orthodontic brackets bonded with different adhesive systems. Eur
J Dent $2017 ; 11: 180-5$.
DOI: $10.4103 /$ ejd.ejd_312_16


surface, thus allowing the infiltration of bacteria, fluids, ions, and even air from the oral cavity. ${ }^{[7-10]}$

This phenomenon, called microleakage, could lead to secondary caries, pulpal inflammation, and the unpleasant "white spot lesions" commonly observed after orthodontic treatment. ${ }^{[1,9-13]}$

One of the causes of orthodontic bracket bond failure is the formation of space between the enamel and the bracket or between the bracket and the adhesive.

In addition to photopolymerization shrinkage, bonding materials are exposed to temperature changes in the oral

cavity caused by the ingestion of food and beverages that could also lead to volumetric changes. These events would produce fatigue in the adhesive joint, which in turn causes microleakage.

Several protocols have been developed to prevent enamel demineralization and reduce bond failure. Despite these efforts, demineralization and bond failure continue to affect the outcome of orthodontic treatment.

The preferred method to study microleakages in vitro continues to be thermal cycling. This method produces a thermal stress on the adhesive joint by recreating extreme temperatures in the teeth similar with those encountered in the oral environment. ${ }^{[9,14]}$

The aim of this study was to assess and compare the stability of different orthodontic adhesive systems at the brackets and enamel junction.

\section{MATERIALS AND METHODS}

A total of sixty healthy mandibular premolars, which had been extracted for orthodontic reasons and immediately placed in normal saline solution $(0.9 \%$ sodium chloride solution), were used in this study.
All the teeth were then etched for $30 \mathrm{~s}$ with $37 \%$ phosphoric acid solution (Ormco Etching Solution, Ormco, Glendora, California) followed by $5 \mathrm{~s}$ of water rinsing and $5 \mathrm{~s}$ of air drying. ${ }^{[11]}$

The prepared teeth were then randomly divided into six groups $(n=10)$ of different adhesive systems, which were assigned a color code [Table 1].

For this study, each orthodontic bracket (3M Victory series; 3M, St Paul, Minnesota) has been bonded to one tooth, particularly to the buccal surface, following manufacturer's instructions. This has been operated by a single operator. Photopolymerization was performed using the Elipar ${ }^{\mathrm{TM}}$ S10 LED Curing Light (3M ESPE, St Paul, Minnesota) at an intensity of $1200 \mathrm{~mW} / \mathrm{cm}^{2}$, for $10 \mathrm{~s}$ on the mesial side and then $10 \mathrm{~s}$ on the distal side.

Two layers of varnish were applied to the teeth based on the color code assigned to each system leaving a $1 \mathrm{~mm}$ margin around the edges of the bracket.

Next, the teeth underwent accelerated aging by means of 2500 cycles of thermal cycling. They were alternately immersed in $5^{\circ} \mathrm{C}$ and $55^{\circ} \mathrm{C}$ baths; each bath lasted $30 \mathrm{~s}$, and transferring the tooth from one bath to the other took $10 \mathrm{~s}$. The teeth were then immersed in $2 \%$ methylene blue for $24 \mathrm{~h}$ away from light before rinsing with water and air dry.

The bracket was then sectioned down the middle in a buccolingual direction using a low-speed diamond saw under continuous irrigation (Isomet ${ }^{\circledR}$ Low-Speed Saw, Springfield, Virginia). Microleakage in each sample was evaluated using a binocular microscope at 40X magnification (Olympus ${ }^{\circledR}$ SZH10, Tokyo, Japan) and an image was taken using a Canon ${ }^{\circledR}$ EOS 700D camera (Tokyo, Japan) connected to the microscope. The image obtained [Figure 1] was analyzed using Image ${ }^{\circledR}$ software version 2006 (Broken Symmetry Software)

\begin{tabular}{|c|c|c|c|c|}
\hline Group & Adhesive & $\begin{array}{l}\text { Number } \\
\text { of teeth }\end{array}$ & Reference & Color \\
\hline I & NeoBond Light Cure Adhesive $\mathrm{Kit}^{\circledR}$ & 10 & Reference: KIT52-800-00 (Dentsply GAC) & Pink \\
\hline II & $\begin{array}{l}\text { Transbond }{ }^{\text {TM }} \text { Plus Self-Etching }+ \text { Transbond }^{\mathrm{TM}} \\
\text { XT Light Cure Adhesive Paste }\end{array}$ & 10 & $\begin{array}{l}\text { Reference: } 712-091(3 \mathrm{M} \text { Unitek) + } \\
\text { reference: N628712 (3M Unitek) }\end{array}$ & $\begin{array}{l}\text { Dark } \\
\text { blue }\end{array}$ \\
\hline III & $\begin{array}{l}\text { Victory V-Slot APC PLUS + Transbond }{ }^{T M} \\
\text { MIP + Scotchbond Universal Etchant }\end{array}$ & 10 & $\begin{array}{l}\text { Reference: 5017-921 (3M Unitek) + reference: } \\
\text { 712-025 (3M Unitek) + reference: } 599816 \text { (3M ESPE) }\end{array}$ & Black \\
\hline IV & Rely-A-Bond Kit ${ }^{\circledR}$ & 10 & Reference: 151913 & Yellow \\
\hline V & Light Cure Orthodontic Adhesive Kit (OptiBond ${ }^{\circledR}$ ) & 10 & Reference: 151913 & Green \\
\hline VI & $\begin{array}{l}\text { Transbond }^{\mathrm{TM}} \text { MIP + Transbond }{ }^{\mathrm{TM}} \text { XT Light Cure } \\
\text { Adhesive Paste + Scotchbond Universal Etchant }\end{array}$ & 10 & $\begin{array}{l}\text { Reference: 712-025 (3M Unitek) + reference: } \\
\text { N628712 (3M Unitek) + reference: } 599816 \text { (3M ESPE) }\end{array}$ & Red \\
\hline
\end{tabular}


which enabled measurements to be made using different tools [Figure 2]. Finally, a score was assigned to the different degrees of microleakage at the bracket-adhesive and adhesive-enamel interfaces in the occlusal and gingival regions of the bracket, as described in Table 2.

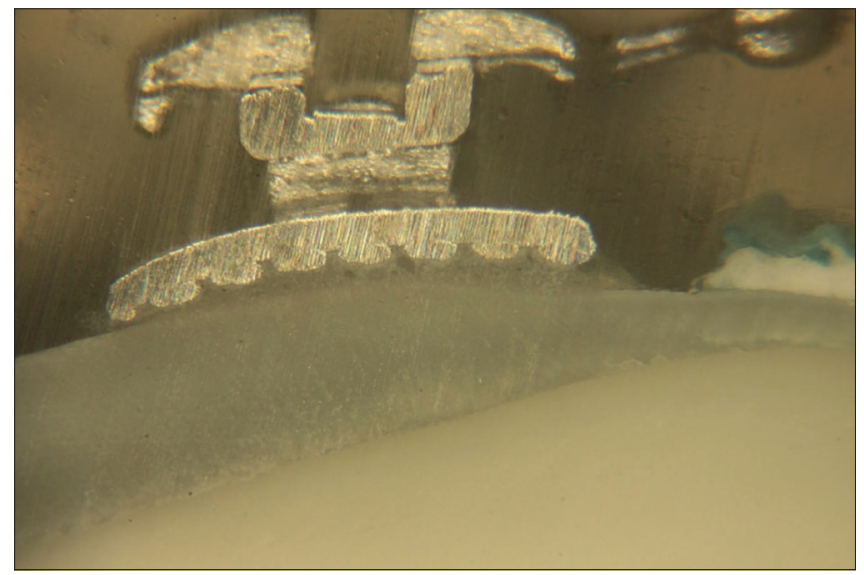

Figure 1: Cemented bracket through binocular microscope $(\times 40)$

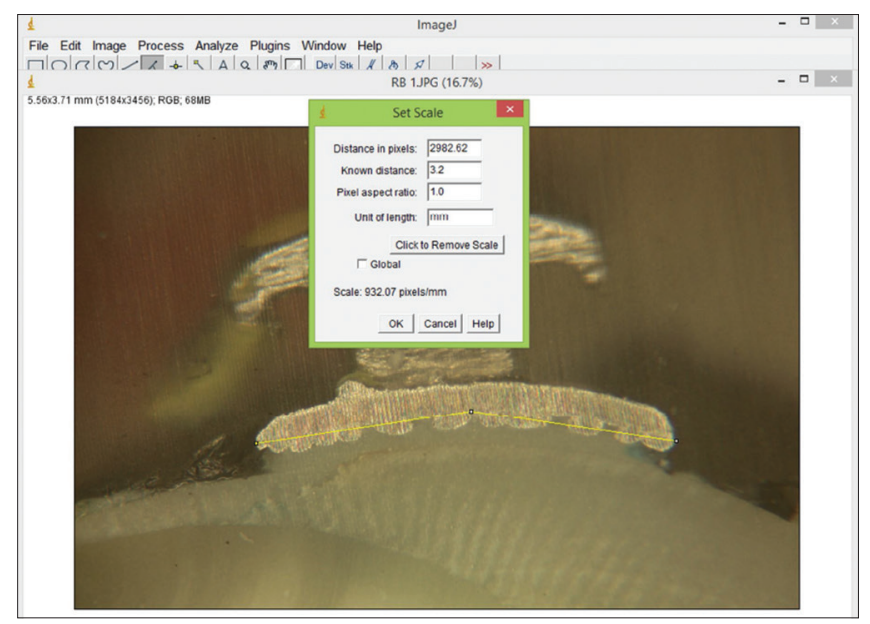

Figure 2: Set scale and measure with Image ${ }^{\circledR}$

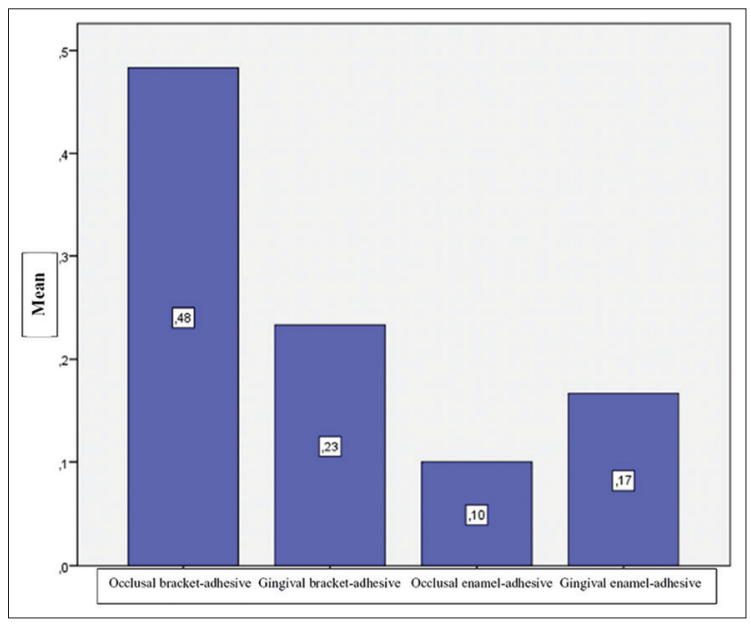

Figure 3: Mean scores according to each interface
Statistical analysis was performed using IBM SPSS ${ }^{\circledR}$ Statistics software (IBM, Armonk, New York).

\section{RESULTS}

A Kruskal-Wallis test yielded a significant difference in the occlusal bracket-adhesive interface among groups depending on the type of adhesive, $P=0.033$. No statistical significant difference was found between the "gingival bracket adhesive," "occlusal adhesive enamel," and "gingival adhesive enamel" groups (respectively, $P=0.197,0.497$, and 0.487 ; see Figure 3). The Score 1 was overrepresented with the "3M Transbond Plus SEP" system (adjusted residual of 2.2), while the Score 0 was overrepresented with the "3M Transbond MIP" adhesive (adjusted residual of 2.7) compared with the respective mean percentages of the Scores 1 and 0 .

Given that the penetration scores were only either 0 or 1 , our data were analyzed by means of four contingency tables for each of the four variables. Results from the two statistical analyses (Kruskal-Wallis and Chi-squared test) were similar. The "occlusal bracket-adhesive" variable alone yielded a statistically significant Chi-squared result $(P=0.034)$ between type of adhesive and score, reflecting the dependence between these two variables.

For the other three variables, as seen in Figure 4, there was no statistically significant difference $(P=0.232$, 0.710 , and 0.742 , respectively).

\section{DISCUSSION}

The goal of this study was to determine whether the type of adhesive material used to bond brackets might play a role in the stability of the bracket-adhesive bonds.

If the stability or "tightness" of a bonding material is compromised, the resulting outcome is microleakage. From an orthodontic perspective, microleakage could

\begin{tabular}{ll} 
Table 2: & Criteria and evaluation of microleakage \\
\hline Score & Criterion \\
\hline 0 & $\begin{array}{l}\text { No dye penetration between bracket-adhesive } \\
\text { or adhesive-enamel interface }\end{array}$ \\
1 & $\begin{array}{l}\text { Penetration of dye limited to } 1 \mathrm{~mm} \text { between bracket } \\
\text { adhesive or adhesive enamel interface }\end{array}$ \\
2 & $\begin{array}{l}\text { Up to } 2 \mathrm{~mm} \text { of dye penetration between bracket adhesive } \\
\text { or adhesive enamel interface } \\
\text { Up to } 3 \mathrm{~mm} \text { of dye penetration between bracket adhesive } \\
\text { or adhesive enamel interface }\end{array}$ \\
\end{tabular}




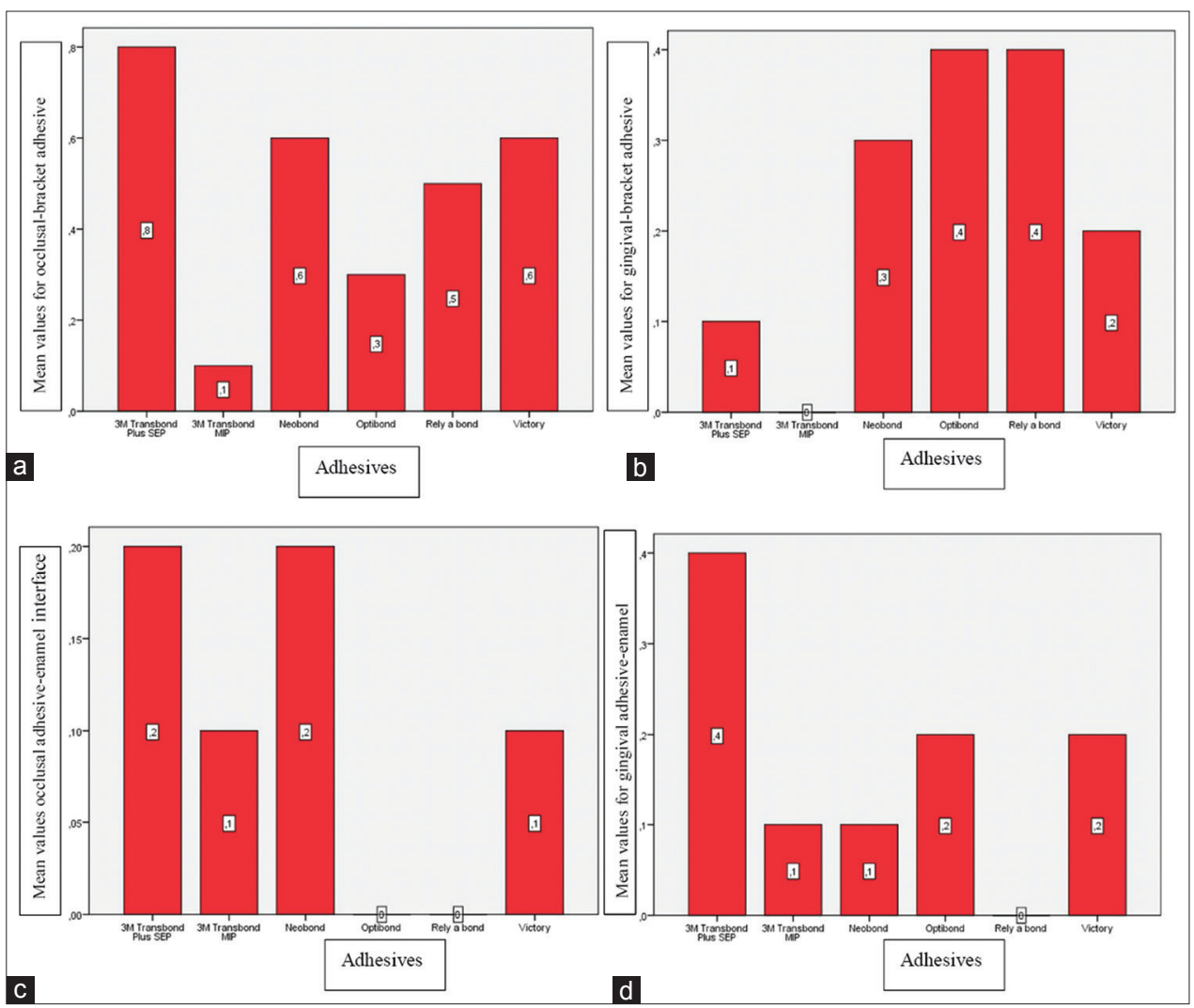

Figure 4: Means adhesive interface scores according to each adhesive. (a) occlusal bracket interface; (b) gingival bracket-adhesive interface; (c) occlusal adhesive-enamel interface; (d) gingival adhesive-enamel interface

very well lead to the formation of white-spot lesions at the "adhesive-enamel" interface. ${ }^{[9,10]}$

These lesions are characterized by enamel decalcification, a known consequence of bacterial plaque's remaining on the enamel surface for a long period of time.

Teeth are daily exposed to temperature variations, due to the ingestion of food and hot and cold drinks and so would be adhesives on teeth. Thus, thermal expansion coefficient of the adhesive must be close to that of dental tissue; otherwise, the tooth and the material will contract and expand at different rates. These repeated movements at different values could cause influx of bacteria-rich fluids of the oral cavity into the spaces formed between the tooth and the filling material. this is known as percolation phenomenon.

Adhesive materials shrink differently while polymerizing. This shrinking factor differs depending on the composition of the adhesive. ${ }^{[1]}$

Transbond Plus Self-Etching, (Group 2), used in this study is a self-etching system. The advantage of this sort of systems is that it reduces the number of steps involved in cementing, thereby reducing procedure time. The etching process is simplified because the etching acid and primer are applied to the dental tissue in one step. ${ }^{[1,2]}$ The "Transbond Plus Self-Etching" system contains phosphoric acid methacrylate, which serves as etching acid and primer. The depth of the demineralized area in the enamel will be the penetration area of the adhesive. Uysal et al. reported that brackets bonded using a self-etching system returned higher microleakage scores than brackets bonded using conventional total etching systems. ${ }^{[1]}$ Their results are consistent with those obtained in our study. As observed in other studies, the gingival margin of the bracket showed a degree of microleakage. However, the occlusal margin of the bracket-adhesive interface showed more microleakage; this was not so in several other studies.

Dye penetration was used for assessing microleakage. It is the easiest and most widely used technique. it is quick, directly measures microleakage, does not react with the hard tissues, is nontoxic, and is cost-effective. ${ }^{[4]}$ The thermal cycling technique have been extensively used in this type of studies. ${ }^{[8,11]}$ 
Thermal expansion is a factor that affects adhesion between the adhesive material and the tooth. The difference in expansion coefficients within the "bracket-adhesive-enamel" complex results in different dimensional changes during temperature variations that may produce fractures, cracks resulting in microleakage. ${ }^{[7]}$

According to the results obtained in our study, the occlusal margin was prone to microleakage at each of the interfaces and with all of the cementing systems, but this difference was not statistically significant. Unlike other studies, the difference in microleakage at the occlusal margin of the "bracket-adhesive" interface was significant.

In a previous study, microleakage scores were higher in the gingival margin of the brackets. Their interpretation of the results was that the differences in scores between the occlusal and gingival margins were due to the tooth morphology, which results in a narrower interface with the adhesive on the gingival side. ${ }^{[7]}$ However, no indication was given of whether the study used maxillary or mandibular premolars. Further, they compared a total etching bonding system against an antibacterial self-etching bonding system on ceramic and metallic brackets. ${ }^{[7]}$ The same results were also obtained by the work of Uysal et al. that compared two adhesive systems on ceramic and metallic brackets. However, they hypothesized that the low microleakage scores at the occlusal margin were due to the adhesive curing method, as they applied the curing light occlusally for $40 \mathrm{s.}^{[1]}$

Looking at our results, the significant difference in the "occlusal bracket-adhesive" variable may be due to the fact that we applied the curing light on the mesial and distal sides for $20 \mathrm{~s}$ each time. Microleakage scores were observed with all the cementing systems. The [Figure 5] shows the comparison between the mean values at the occlusal and gingival interface for each adhesive brands.

Microleakage was present in all groups. We observed a significant difference in the "occlusal bracket adhesive" group. In the "gingival bracket adhesive," "occlusal adhesive enamel," and "gingival adhesive enamel" groups, there was no significant difference. The adhesive that returned the lowest microleakage score was Group 6, "Transbond MIP;" conversely, Group 2, "Transbond Plus Self-Etching," returned the highest microleakage scores. Group 2

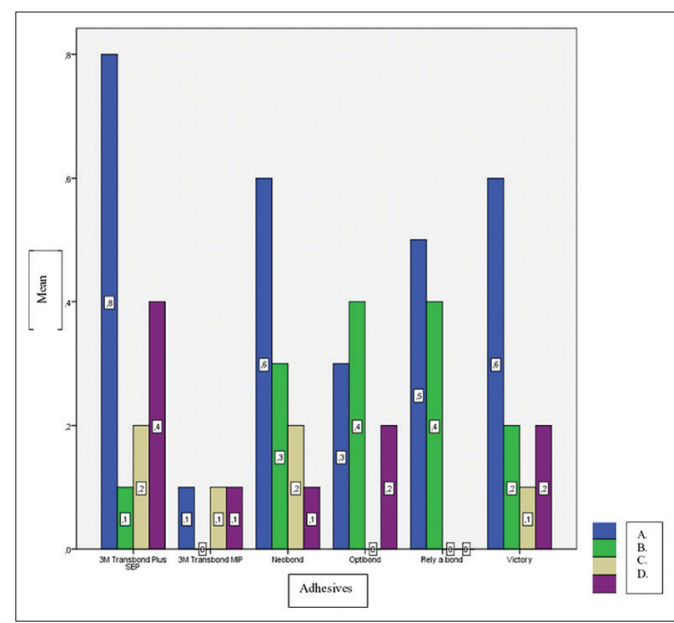

Figure 5: Mean scores of the different adhesive brands at each occlusal and gingival interface

was a self-etching system whereas Group 6 was a conventional etching system. We can conclude that enamel bonding using conventional etching results in greater, better-quality adhesion.

\section{Financial support and sponsorship}

Nil.

\section{Conflicts of interest}

There are no conflicts of interest.

\section{REFERENCES}

1. Uysal T, Ulker M, Ramoglu SI, Ertas H. Microleakage under metallic and ceramic brackets bonded with orthodontic self-etching primer systems. Angle Orthod 2008;78:1089-94.

2. Ramesh Kumar KR, Shanta Sundari KK, Venkatesan A, Chandrasekar $\mathrm{S}$. Depth of resin penetration into enamel with 3 types of enamel conditioning methods: A confocal microscopic study. Am J Orthod Dentofacial Orthop 2011;140:479-85.

3. Iijima M, Ito S, Yuasa T, Muguruma T, Saito T, Mizoguchi I. Bond strength comparison and scanning electron microscopic evaluation of three orthodontic bonding systems. Dent Mater J 2008;27:392-9.

4. Yagci A, Uysal T, Ulker M, Ramoglu SI. Microleakage under orthodontic brackets bonded with the custom base indirect bonding technique. Eur J Orthod 2010;32:259-63.

5. Cal-Neto JP, Miguel JA. Scanning electron microscopy evaluation of the bonding mechanism of a self-etching primer on enamel. Angle Orthod 2006;76:132-6.

6. Toodehzaeim MH, Yassaei S, Karandish M, Farzaneh S. In vitro evaluation of microleakage around orthodontic brackets using laser etching and Acid etching methods. J Dent (Tehran) 2014;11:263-9.

7. Arhun N, Arman A, Cehreli SB, Arikan S, Karabulut E, Gülsahi K. Microleakage beneath ceramic and metal brackets bonded with a conventional and an antibacterial adhesive system. Angle Orthod 2006;76:1028-34.

8. Vicente A, Ortiz AJ, Bravo LA. Microleakage beneath brackets bonded with flowable materials: Effect of thermocycling. Eur J Orthod 2009;31:390-6.

9. Courson F. Adhesive Techniques in Pediatric Odontology. In vitro Evaluation of Sealing Biomaterials Used to Obturate Permanent Teeth. Comparative Study of Adhesion Mechanisms from Temporary to Definite Tooth. Montrouge, France, Thesis; 2003.

10. Buyuk SK, Cantekin K, Demirbuga S, Ozturk MA. Are the low-shrinking composites suitable for orthodontic bracket bonding? Eur J Dent 2013;7:284-8. 
11. Canbek K, Karbach M, Gottschalk F, Erbe C, Wehrbein H. Evaluation of bovine and human teeth exposed to thermocycling for microleakage under bonded metal brackets. J Orofac Orthop 2013;74:102-12.

12. Pashley DH, Tay FR, Breschi L, Tjäderhane L, Carvalho RM, Carrilho $\mathrm{M}$, et al. State of the art etch-and-rinse adhesives. Dent Mater
2011;27:1-16

13. Kidd EA. Microleakage in relation to amalgam and composite restorations. A laboratory study. Br Dent J 1976;141:305-10.

14. Alkis H, Turkkahraman $\mathrm{H}$, Adanir N. Microleakage under orthodontic brackets bonded with different adhesive systems. Eur J Dent 2015;9:117-21. 